\title{
Acellular dermal matrix in implant-based immediate breast reconstructions: a comparison of prepectoral and subpectoral approach
}

\author{
Mihir Navin Chandarana, Sadaf Jafferbhoy, Sekhar Marla, Soni Soumian, Sankaran Narayanan \\ Breast Unit, Department of General Surgery, University Hospital of North Midlands, Stoke-on-Trent, UK \\ Contributions: (I) Concept and design: All authors; (II) Administrative support: All authors; (III) Provision of study material or patients: All authors; \\ (IV) Collection and assembly of data: All authors; (V) Data analysis and interpretation: All authors; (VI) Manuscript writing: All authors; (VII) Final \\ approval of manuscript: All authors. \\ Correspondence to: Mr. Sankaran Narayanan. Department of General Surgery, University Hospital North Midlands, Trust Headquarters Building, The \\ Royal Stoke University Hospital, Stoke on Trent, ST4 6QG, UK. Email: Sankaran.Narayanan@uhnm.nhs.uk.
}

\begin{abstract}
Background: Implant-based immediate breast reconstruction (IBR) is the most common technique for post-mastectomy reconstructions in the United Kingdom (UK). Subpectoral implant placement is the conventional method of reconstruction. Placement of implant in the subcutaneous pocket covered by an acellular dermal matrix (ADM) is a relatively recent approach. We report a comparative analysis of prepectoral versus subpectoral approach for implant-based IBR from a single institution in the UK.

Methods: Retrospective analysis from a prospectively maintained data was conducted from January 2015 to May 2017 including all patients who underwent a mastectomy with immediate implant-based IBR in a single breast unit. The demographic details, operative details, immediate and delayed complications were recorded. Specific complications recorded were infection, seroma, unplanned readmission and loss of implant. Factors affecting complication rates and implant loss were analyzed.

Results: One hundred and fifty-four reconstructions were included in the analysis with a median followup of 11.8 months. Mean age of the cohort was 50 years with a mean BMI of $26.09 \mathrm{~kg} / \mathrm{m}^{2}$. Major implantrelated complication rate was $12.3 \%$ with an implant loss rate of $7.8 \%$. Age more than 50 years $(\mathrm{P}=0.037)$ and bilateral reconstructions $(\mathrm{P}=0.0001)$ had significant impact on complication rate, on multivariate analysis. Patients with bilateral implants had a significantly higher implant loss rate $(\mathrm{P}=0.0001)$. Implant loss rates in the prepectoral group (4.2\%) and subpectoral group (10.8\%) were not statistically significant $(\mathrm{P}=0.29)$.

Conclusions: Prepectoral and subpectoral techniques of IBR have comparable outcomes. Studies reporting on long-term outcomes are planned.
\end{abstract}

Keywords: Acellular dermis; breast implants; breast neoplasms

Submitted Feb 13, 2018. Accepted for publication Mar 05, 2018.

doi: $10.21037 /$ gs.2018.03.05

View this article at: http://dx.doi.org/10.21037/gs.2018.03.05

\section{Introduction}

The last decade has seen a steady rise in mastectomy rates in the United Kingdom (UK) (1) with the numbers of risk reducing mastectomies having more than doubled in the UK and United States (2). In parallel with increasing mastectomy rates, there has been a rise in immediate and delayed breast reconstructions. In the UK, implant-based reconstruction has become the most preferred approach for immediate breast reconstruction (IBR) and has increased from $30 \%$ in 2007 to $54 \%$ in 2014 (3).

The introduction of acellular dermal matrix (ADM) by Salzberg et al. in 2016 as an adjunct in subpectoral reconstructions has led to a significant increase in its use for IBR and his series of more than 1,500 reconstructions 
has demonstrated acceptable results with low capsular contracture rates (4). There are various ADMs currently in use, the commonest being Alloderm ${ }^{\circledR}$ (LifeCell Corp., Branchburg, New Jersey, USA), Strattice ${ }^{\circledR}$ (Lifecell, Branchburg, New Jersey, USA), Surgimend ${ }^{\circledR}$ (TEI Biosciences, Boston, Massachusetts, USA), Native ${ }^{\circledR}$ (MBP Biologics, Neustadt-Glewe, Germany, licence holder Decomed, Marcon, Venezia, Italy) and Veritas ${ }^{\circledR}$ (Synovis Surgical Innovations, St. Paul, MN, USA). The use of $\mathrm{ADM}$ has led to reduction in capsular contracture rates and acceptable perioperative outcomes (4-6). There is no published evidence with regard to the superiority of any individual $\mathrm{ADM}$ over the other.

Implant placement in the subpectoral pocket has been the conventional technique of implant-based IBR. Subpectoral implant along with ADM provides complete implant coverage, a larger pocket and control of inframammary fold. Other advantages are minimal implant visibility and reduced rippling. However the morbidity associated with pectoralis muscle detachment, animation deformity and post-operative pain remain a matter of concern $(7,8)$.

Prepectoral implant placement with complete coverage of the implant with ADM avoids the detachment of the pectoralis major muscle. Braxon ${ }^{\circledR}$ (MBP Biologics, NeustadtGlewe, Germany, licence holder Decomed, Marcon, Venezia, Italy) is a novel ADM used for prepectoral implantbased IBR. It is a $0.6-\mathrm{mm}$ thick mesh derived from porcine dermis, available as a pre-shaped mesh to be wrapped around the implant ex vivo. As this is a relatively new technique, there is paucity of data regarding its role in IBR. Two multicentre reports on prepectoral implant-based IBR using Braxon $^{\circledR}$ ADM have been published recently with outcomes comparable to National data from the UK (9-12). However, long-term results are awaited. To our knowledge, there has been no data in literature comparing single stage subpectoral and prepectoral implant-based IBR using ADM. We report on these outcomes from a single institution in the UK.

\section{Methods}

A retrospective analysis of prospectively collected data was conducted. All consecutive patients undergoing mastectomy with an implant-based IBR performed by three oncoplastic breast surgeons at University Hospitals of North Midlands from $1^{\text {st }}$ January 2015 to $31^{\text {st }}$ May 2017 with a minimum follow-up of 3 months were included in the analysis. Patients with reconstructions using a tissue expander or any autologous form of reconstructions with or without an implant were excluded. Demographic factors, tumour characteristics and perioperative outcomes were evaluated. Perioperative outcomes studied included early and delayed complications, unplanned readmission, return to theatre and implant loss. Early complications were defined as any complications occurring up to 90 days after reconstructive surgery. Delayed complications were defined as those recorded from day 91 to one year after surgery. All complications with Clavien-Dindo grade III or higher were classified as major complications (13). Removal of implant was classified as implant loss. Re-exploration requiring an exchange of implant or placement of a tissue expander was classified as a major complication. Age, body mass index (BMI), laterality, indication for surgery, smoking, T-stage, nodal status, implant position (prepectoral versus subpectoral), chemotherapy and adjuvant radiation therapy were the factors assessed for their impact on complication rates and implant loss.

\section{Statistical analysis}

Data was presented as mean, median or frequency (percentage) as appropriate. Group comparisons were made using independent $t$-test or Mann Whitney $\mathrm{U}$ test for continuous variables and using Chi-square test or Fischer's exact test for categorical variables. Multivariate analysis was performed using logistic regression. A $\mathrm{P}$ value of less than 0.05 was taken as significant. Statistical analysis was carried out using SPSS version 21 for Windows SPSS 21.0 software (SPSS Inc., Chicago, IL USA).

\section{Results}

One hundred and thirty patients had mastectomy with implant-based IBR from $1^{\text {st }}$ January 2015 to $31^{\text {st }}$ May 2017 out of which 24 patients $(18.5 \%)$ had a bilateral procedure. Thus, the analysis included 154 procedures. Demographic details, tumour characteristics and treatment details of the patients are shown in Table 1 . Mean age of the cohort was 50 years with a mean BMI of $26.09 \mathrm{~kg} / \mathrm{m}^{2}$. Forty six percent $(71 / 154)$ of the procedures were prepectoral implant reconstructions and 54\% (83/154) were subpectoral. The two groups were comparable with regards to demographic and treatment characteristics. All the prepectoral reconstructions were performed with the aid of Braxon ${ }^{\circledR}$. Native ${ }^{\circledR}$ and Strattice ${ }^{\circledR}$ were the ADMs used for $83 \%$ of the subpectoral reconstructions (Figures 1,2). The largest implant size for the prepectoral group was $520 \mathrm{cc}$ 
Table 1 Patient, tumour and treatment factors

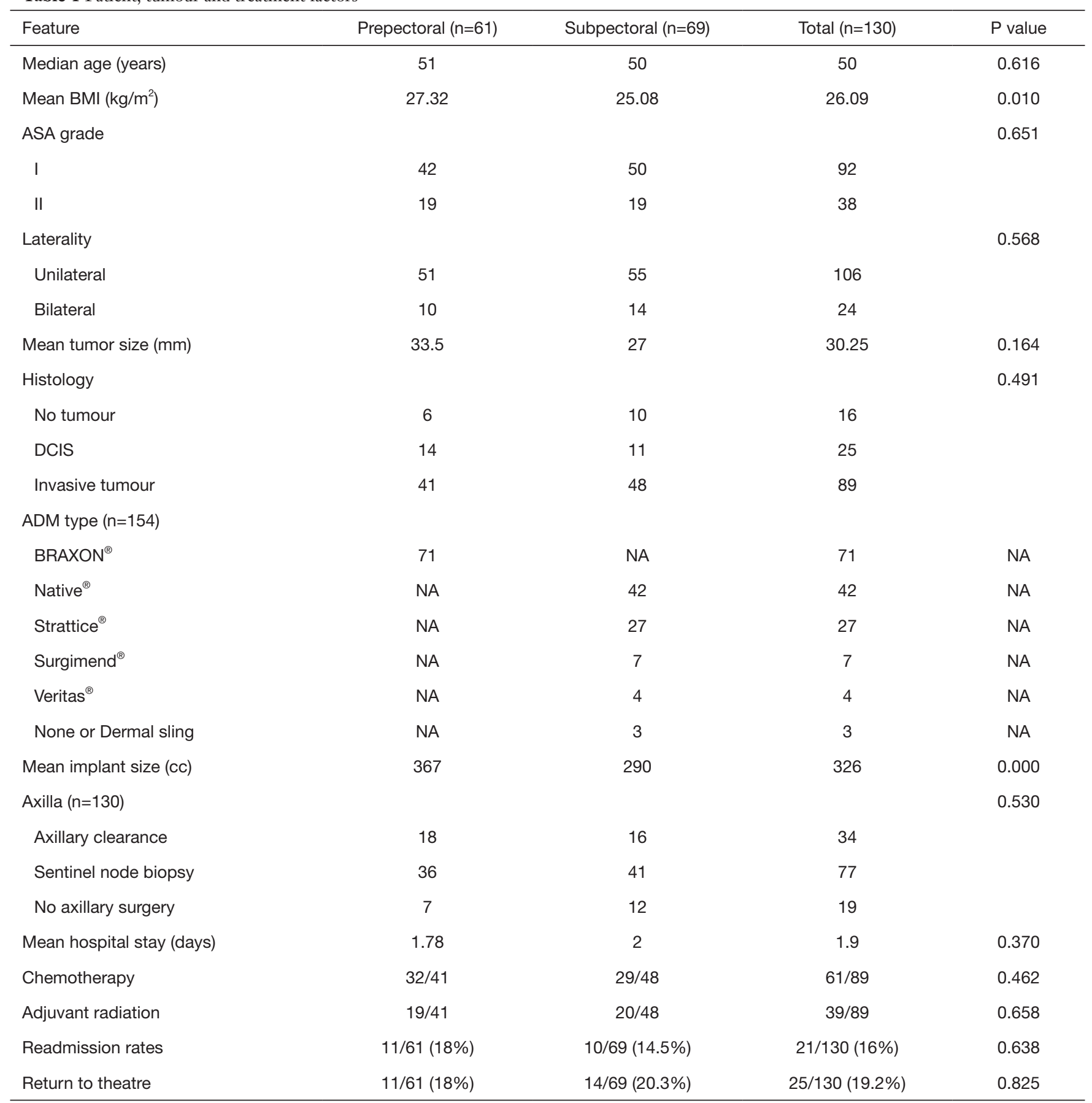

BMI, body mass index; ASA, American Society of Anesthesiologists; DCIS, ductal carcinoma in situ; ADM, acellular dermal matrix.

(range, 165-520 cc) and that for subpectoral group was $560 \mathrm{cc}$ (range, $105-560 \mathrm{cc}$ ). Of the patients treated for invasive tumours, $69 \%$ received chemotherapy and $44 \%$ patients received adjuvant radiation therapy.

Twenty-nine patients (22\%) had a major complication.
Of these, 12 patients had a post-operative haematoma and 1 patient had a post-operative pulmonary embolism. Therefore, 16 patients $(12.3 \%)$ had a major implantrelated complication. Of the 16 patients, 7 (5.4\%) had a prepectoral procedure and $9(6.9 \%)$ had a subpectoral 

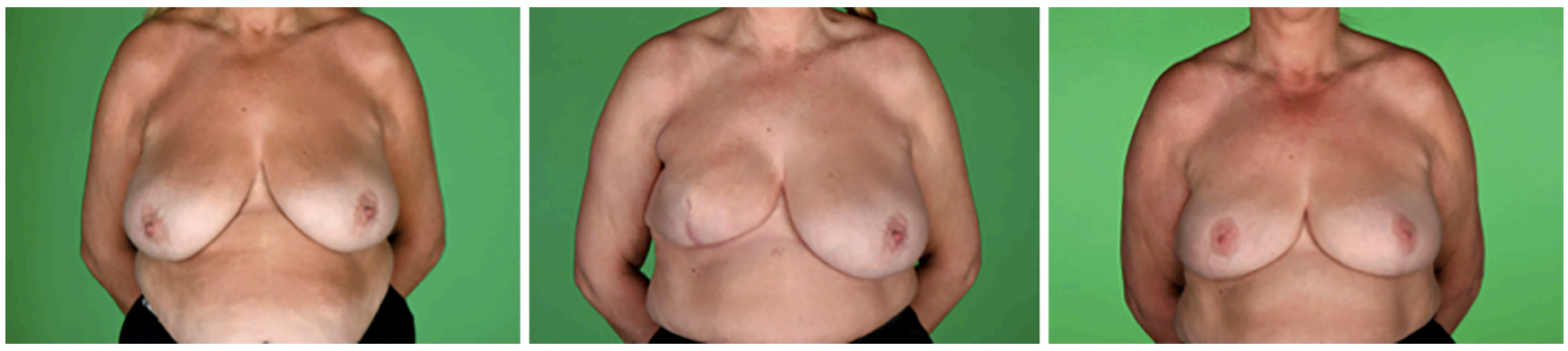

Figure 1 Right skin-sparing mastectomy with subpectoral implant. Pre-operative, 3-month post-operative and 21-month post-operative follow-up (left breast reduction and bilateral nipple reconstruction with tattooing done).
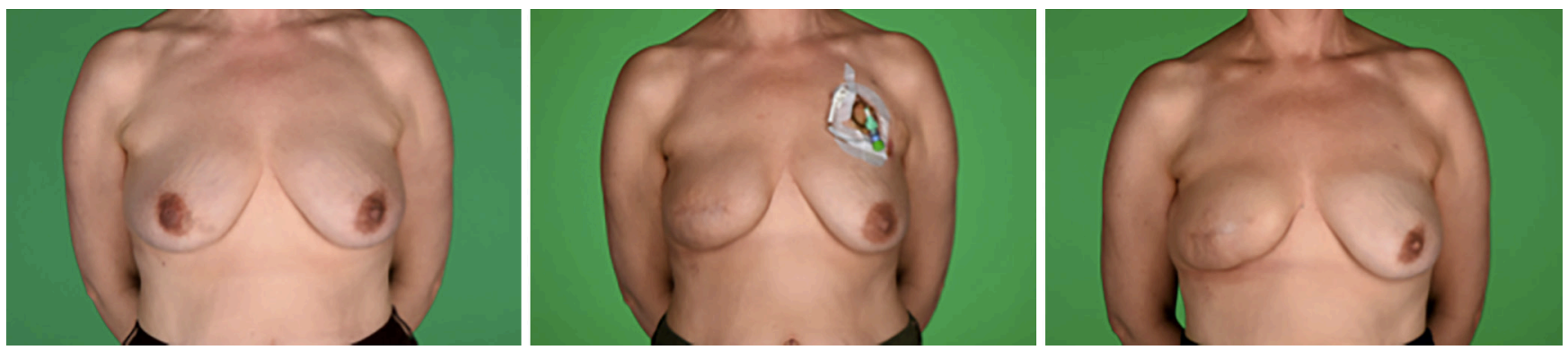

Figure 2 Right skin-sparing mastectomy with prepectoral implant. Pre-operative, 5-month and 12-month post-operative follow-up.

implant (Table 2). Unplanned readmission rate was $16 \%$ and return to theatre rate was $19 \%$ for all complications. Seven patients [(7/130 (5.4\%)] needed implant removal secondary to complications. Five of the seven patients had bilateral procedures, thus the implant loss rate was $7.8 \%$ (12/154 procedures). Of the 12 implants removed, three were prepectoral and nine were subpectoral. This gives an implant loss rate of $4.2 \%$ in the prepectoral group and $10.8 \%$ in the subpectoral group. Twenty-nine patients (22\%) had minor complications-mainly seroma needing aspiration or wound redness. Thirteen patients (10\%) developed delayed complications (Table 2). None of the patients with delayed complications lost their implants. Forty-one patients $(31.5 \%)$ underwent or were planned for additional procedures like lipomodelling, nipple reconstructions or contralateral breast reduction.

Median follow-up of the entire group was 11.8 months. It was 9.8 months for the prepectoral group and 19.6 months for the subpectoral group. On analysis of the factors with a potential to impact on complication and implant loss rates, age more than 50 years $(\mathrm{P}=0.037)$ and bilateral reconstructions $(\mathrm{P}=0.0001)$ were associated with significantly more early complications on univariate and multivariate analysis. Bilateral implant-based IBR was the only factor significantly associated with loss of implant $(\mathrm{P}=0.017)$. There was no statistical difference in complication rate $(\mathrm{P}=0.52)$ or loss of implant $(\mathrm{P}=0.29)$ between prepectoral and subpectoral groups.

\section{Discussion}

Mastectomy rates have increased over the last decade in the UK accompanied by a rise in the reconstruction rates with more than half of IBR being implant-based (3). Our study was a comparative analysis of subpectoral and prepectoral implant-based reconstructions using ADM based on data that was prospectively collected. To our knowledge, there have been no reports in literature comparing single stage subpectoral and prepectoral procedures using ADM.

Berna et al. first reported their outcomes in a series of 19 patients with 25 prepectoral reconstructions using Braxon ${ }^{\circledR}$. In their series, three patients had implant loss. Interestingly, all the major complications occurred in patients with a thicker variety of Braxon ${ }^{\circledR}$ measuring $0.9 \mathrm{~mm}$. The shortterm follow-up demonstrated adequate clinical and aesthetic outcomes (14). There have been two multicentre reports on feasibility and short-term outcomes of prepectoral reconstructions using Braxon ${ }^{\circledR}$. Vidya et al. have reported on 100 reconstructions from centres in Europe with an 
Table 2 Major complications-early and delayed

\begin{tabular}{|c|c|c|c|}
\hline Complication details $(n=130)$ & Prepectoral & Subpectoral & Total \\
\hline \multicolumn{4}{|l|}{ Early complications } \\
\hline Haematoma & 7 & 5 & 12 \\
\hline SSI & 4 & 5 & 9 \\
\hline Skin necrosis & 0 & 4 & 4 \\
\hline Seroma & 1 & 0 & 1 \\
\hline Wound dehiscence & 1 & 0 & 1 \\
\hline Redness & 1 & 0 & 1 \\
\hline Others (PE) & 0 & 1 & 1 \\
\hline Total & 14 & 15 & 29 \\
\hline \multicolumn{4}{|l|}{ Delayed complications } \\
\hline Capsular contracture & 1 & 5 & 6 \\
\hline Skin necrosis & 3 & 0 & 3 \\
\hline Skin redness & 1 & 0 & 1 \\
\hline Wound dehiscence & 1 & 0 & 1 \\
\hline SSI & 0 & 1 & 1 \\
\hline Others & 1 & 0 & 1 \\
\hline Total & 7 & 6 & 13 \\
\hline
\end{tabular}

PE, pulmonary embolism; SSI, surgical site infection.

implant loss rate of two percent and satisfactory cosmetic outcomes (9). Jafferbhoy et al. have reported a multicentre study on 78 reconstructions from the UK in which the implant loss rate was $10 \%$ with short-term outcomes comparable to the National Mastectomy and Reconstruction Audit (NMBRA) and the recently conducted Implant-based Breast Reconstruction evaluation audit (IBRA) (10-12). Other studies have looked at two-stage post-mastectomy reconstructions with a tissue expander comparing prepectoral versus subpectoral placement (15-17). Casella et al. have reported on similar outcomes comparing prepectoral and subpectoral single-stage IBR using a synthetic titanized mesh $\left(\right.$ TiLOOP $\left.^{\circledR}\right)$ in a series of 73 mastectomies with an implant loss in one patient and a complication rate of less than $10 \%$ in each group. However, the selection criteria were quite stringent and more than $70 \%$ of patients had stage 0 or IA tumours (18).

In our series, the overall outcomes between the two groups were comparable. Twelve patients had a post-operative haematoma, which is not a reconstruction related complication. The overall implant loss rates of $7.8 \%$ were similar to National data published in NMBRA (9\%) and the recently conducted IBRA study $(8.9 \%)(11,12)$. Although the implant loss rates were lower in the prepectoral group (4.2\%) as compared to subpectoral group (10.8\%), the difference was not found to be statistically significant. This might be due to the small sample size and lower event rates of the cohort. One could argue that the readmission rates (16\%) and return to theatre rates (19\%) were higher than expected, which were comparable between the two groups (Table 1). We had a relatively low threshold for readmission and exploration, which could have contributed in salvaging some reconstructions. Also, these were comparable to data from NMBRA and the recently conducted IBRA studies $(11,12)$.

Our study included consecutive patients operated in breast unit from a single institution. The treatment protocols with regard to the operative technique, peri-operative antibiotic use and post-operative management were uniform, which might not be the case in multicentre studies. A randomised controlled trial (RCT) would be ideal to compare the outcomes of prepectoral and subpectoral reconstructions. However, its feasibility should be assessed before embarking up on a RCT. In absence of evidence from a RCT, a wellconducted prospective analysis would be the best evidence available. The median follow-up in the prepectoral group was 9.8 months and a longer follow-up would be necessary to assess rippling and impact of adjuvant radiation therapy.

Our study does have some drawbacks. It is a retrospective analysis of a prospective database and will have the disadvantages of a retrospective study (19). Implant loss rates were significantly higher in bilateral implant-based reconstructions. The reason for this is not clear and needs further evaluation. Patient-related quality of life outcome measures and post-operative pain scores need to be evaluated, as these are important parameters for comparison of prepectoral versus subpectoral reconstructions.

\section{Conclusions}

Our study has shown comparable results between prepectoral and subpectoral technique of implant-based IBR. Age more than 50 years and bilateral reconstructions were factors significantly associated with major complications. Bilateral reconstructions had significantly higher implant loss rates. Further studies reporting long-term outcomes are planned.

\section{Acknowledgements}

None. 


\section{Footnote}

Conflicts of Interest: The authors have no conflicts of interest to declare.

Ethical Statement: As no new or any experimental treatment was offered to the patients, an ethics committee approval was not required. Informed consent was obtained from all patients to include their treatment and management details into the hospital database for audit and governance purposes. Patients consented separately for clinical photographs to be taken (data anonymised) for hospital records, teaching, presentation in scientific meetings and publication in scientific journals.

\section{References}

1. Kummerow KL, Du L, Penson DF, et al. Nationwide trends in mastectomy for early-stage breast cancer. JAMA Surg 2015;150:9-16.

2. Neuburger J, MacNeill F, Jeevan R, et al. Trends in the use of bilateral mastectomy in England from 2002 to 2011: retrospective analysis of hospital episode statistics. BMJ Open 2013;3:e003179.

3. Mennie JC, Mohanna PN, O’Donoghue JM, et al. National trends in immediate and delayed postmastectomy reconstruction procedures in England: A seven-year population-based cohort study. Eur J Surg Oncol 2017;43:52-61.

4. Salzberg CA, Ashikari AY, Berry C, et al. Acellular Dermal Matrix-Assisted Direct-to-Implant Breast Reconstruction and Capsular Contracture: A 13-Year Experience. Plast Reconstr Surg 2016;138:329-37.

5. Cabalag MS, Rostek M, Miller GS, et al. Alloplastic adjuncts in breast reconstruction. Gland Surg 2016;5:158.

6. Vardanian AJ, Clayton JL, Roostaeian J, et al. Comparison of Implant-Based Immediate Breast Reconstruction with and without Acellular Dermal Matrix. Plast Reconstr Surg 2011;128:403e-10e.

7. Spear SL, Seruya M, Clemens MW, et al. Acellular Dermal Matrix for the Treatment and Prevention of Implant-Associated Breast Deformities: Plast Reconstr Surg 2011;127:1047-58.

8. Hammond DC, Schmitt WP, O'Connor EA. Treatment of Breast Animation Deformity in Implant-Based Reconstruction with Pocket Change to the Subcutaneous Position. Plast Reconstr Surg 2015;135:1540-4.

9. Vidya R, Masià J, Cawthorn S, et al. Evaluation of the effectiveness of the prepectoral breast reconstruction with
Braxon dermal matrix: First multicenter European report on 100 cases. Breast J 2017;23:670-6.

10. Jafferbhoy S, Chandarana M, Houlihan M, et al. Early multicentre experience of pre-pectoral implant based immediate breast reconstruction using Braxon®. Gland Surg 2017;6:682-8.

11. Browne J, Pereira J, Caddy C, et al. British Association of Plastic, Reconstructive and Aesthetic Surgeons. [cited 2017 Sep 17]; Available online: http://promesi.med.auth. gr/mathimata/clin-audi-supp-prog-mast-brea-reco-2011rep1.pdf

12. ABS Conference 2017 [Internet]. Available online: http:// associationofbreastsurgery.org.uk/events/previousmeetings/2017/abs-conference-2017/\#

13. Dindo D, Demartines N, Clavien PA. Classification of surgical complications: a new proposal with evaluation in a cohort of 6336 patients and results of a survey. Ann Surg 2004;240:205-13.

14. Berna G, Cawthorn SJ, Papaccio G, et al. Evaluation of a novel breast reconstruction technique using the Braxon $\AA$ acellular dermal matrix: a new muscle-sparing breast reconstruction. ANZ J Surg 2017;87:493-8.

15. Schnarrs RH, Carman CM, Tobin C, et al. Complication rates with human acellular dermal matrices: retrospective review of 211 consecutive breast reconstructions. Plast Reconstr Surg Glob Open 2016;4:e1118.

16. Zhu L, Mohan AT, Abdelsattar JM, et al. Comparison of subcutaneous versus submuscular expander placement in the first stage of immediate breast reconstruction. J Plast Reconstr Aesthet Surg 2016;69:e77-86.

17. Bettinger LN, Waters LM, Reese SW, et al. Comparative Study of Prepectoral and Subpectoral Expander-Based Breast Reconstruction and Clavien IIIb Score Outcomes. Plast Reconstr Surg Glob Open 2017;5:e1433.

18. Casella D, Bernini M, Bencini L, et al. TiLoop® Bra mesh used for immediate breast reconstruction: comparison of retropectoral and subcutaneous implant placement in a prospective single-institution series. Eur J Plast Surg 2014;37:599-604.

19. Sedgwick P. Retrospective cohort studies: advantages and disadvantages. BMJ 2014;348:g1072.

Cite this article as: Chandarana MN, Jafferbhoy S, Marla S, Soumian S, Narayanan S. Acellular dermal matrix in implantbased immediate breast reconstructions: a comparison of prepectoral and subpectoral approach. Gland Surg 2018;7(Suppl 1):S64-S69. doi: 10.21037/gs.2018.03.05 